\title{
Tacrolimus for the Treatment of Fistulas in Patients With Crohn's Disease: A Randomized, Placebo-Controlled Trial
}

\author{
WILLIAM J. SANDBORN,* DANIEL H. PRESENT, ${ }^{\dagger}$ KIM L. ISAACS,§ DOUGLAS C. WOLF,॥ \\ EUGENE GREENBERG, " STEPHEN B. HANAUER, \# BRIAN G. FEAGAN, ** LLOYD MAYER, ${ }^{\dagger}$ \\ THERESE JOHNSON,* JOSEPH GALANKO,§ CHRISTOPHER MARTIN,§ and ROBERT S. SANDLER, $\$$ \\ FOR THE CROHN'S AND COLITIS FOUNDATION OF AMERICA CLINICAL ALLIANCE \\ * Mayo Clinic, Rochester, Minnesota; ${ }^{\dagger}$ Mount Sinai School of Medicine, New York, New York; §Division of Digestive Diseases and Nutrition, \\ University of North Carolina at Chapel Hill, Chapel Hill, North Carolina; "Atlanta Gastroenterology Associates, Atlanta, Georgia; "Carle Clinic \\ Association, Urbana, Illinois; \#University of Chicago, Chicago, Illinois; and **University of Western Ontario, London, Ontario, Canada
}

Background \& Aims: This study determined the effectiveness of tacrolimus for the treatment of Crohn's disease fistulas. Methods: The study was a randomized, double-blind, placebo-controlled, multicenter clinical trial. Forty-eight patients with Crohn's disease and draining perianal or enterocutaneous fistulas were randomized to treatment with oral tacrolimus $0.2 \mathrm{mg}$. $\mathrm{kg}^{-1} \cdot$ day $^{-1}$ or placebo for 10 weeks. The primary outcome measure was fistula improvement as defined by closure of $\geq 50 \%$ of particular fistulas that were draining at baseline and maintenance of that closure for at least 4 weeks. A secondary outcome measure was fistula remission as defined by closure of all fistulas and maintenance of that closure for at least 4 weeks. Results: Forty-three percent of tacrolimus-treated patients had fistula improvement compared with $8 \%$ of placebo-treated patients $(P=0.004)$. Ten percent of tacrolimus-treated patients had fistula remission compared with $8 \%$ of placebo-treated patients $(P=0.86)$. Adverse events significantly associated with tacrolimus, including headache, increased serum creatinine level, insomnia, leg cramps, paresthesias, and tremor, were managed with dose reduction. Conclusions: Oral tacrolimus $0.2 \mathrm{mg} \cdot \mathrm{kg}^{-1} \cdot$ day $^{-1}$ is effective for fistula improvement, but not fistula remission, in patients with perianal Crohn's disease. Adverse events associated with tacrolimus can be managed by dose reduction. Lower doses of tacrolimus should be evaluated.

$\mathrm{F}$ istulas occur at some point during the lifetime disease course in $20 \%-40 \%$ of patients with Crohn's disease. ${ }^{1-4}$ Surgical treatments consist of fistulotomy, placement of noncutting setons, and bowel resection. 5,6 Medical therapies reported to be effective in uncontrolled trials include metronidazole, ${ }^{7,8}$ 6-mercaptopurine, 9 and cyclosporine. ${ }^{10-12}$ However, controlled trials are rare. A meta-analysis of placebo-controlled trials of azathioprine and 6-mercaptopurine suggested efficacy in the subgroup of patients with fistulas, ${ }^{13}$ and a single placebo-controlled trial showed that infliximab is effective for the short-term closure ( 4 weeks) of enterocutaneous and perianal fistulas. ${ }^{14}$

Tacrolimus inhibits the production of interleukin 2 by T-helper cells. ${ }^{15,16}$ Uncontrolled studies have suggested that tacrolimus at doses of $0.1-0.3 \mathrm{mg} \cdot \mathrm{kg}^{-1} \cdot$ day ${ }^{-1}$ may be of benefit in patients with Crohn's disease fistulas. ${ }^{17-20}$ Based on these preliminary results, we conducted a 10-week trial in which patients with Crohn's disease and open draining fistulas received oral tacrolimus $0.2 \mathrm{mg} \cdot \mathrm{kg}^{-1} \cdot$ day $^{-1}$ or placebo.

\section{Patients and Methods}

\section{Selection of Patients}

The study was performed between June 14, 1999, and January 8, 2000. Eligible patients were at least 12 years of age and had Crohn's disease and 1 or more open draining enterocutaneous fistulas (perianal or abdominal wall) that had not closed despite previous treatment with at least 1 antibiotic. The following patients were not eligible: those immediately in need of surgery for active gastrointestinal bleeding, peritonitis, intestinal obstruction, or intra-abdominal abscess requiring surgical drainage; those with local or systemic infection (including perianal abscess); those with symptoms of bowel obstruction within the preceding 6 months (confirmed by findings of dilation of the bowel proximal to the stricture on barium x-ray or an inability to traverse a stricture at endoscopy) that had not been surgically corrected; those with a planned inpatient hospitalization during the study; those with other clinically important active diseases (such as renal or hepatic disease); and those with open draining fistulas that

\footnotetext{
Abbreviations used in this paper: CCFA, Crohn's and Colitis Foundation of America; IBDQ, Inflammatory Bowel Disease Questionnaire.

(C) 2003 by the American Gastroenterological Association 0016-5085/03/\$30.00 doi:10.1016/S0016-5085(03)00877-1
} 
only involved the vagina, bladder, or bowel (enteroenteric fistulas). Patients with a history of dysplasia of the colon (includes flat dysplasia or sporadic adenomatous polyps) or any cancer within 5 years or clinically significant hematologic values (not specifically defined) or biochemical values (serum creatinine concentration $>1.5$ times the upper limit of normal or alkaline phosphatase, aspartate aminotransferase, or alanine aminotransferase concentrations $>2.5$ times the upper limit of normal) were also ineligible, as were pregnant or breast-feeding women and those with a history of drug or alcohol abuse within the preceding 6 months. The decision of whether surgical consultation, examination under anesthesia, and abdominal computed tomography scans were required to exclude perianal abscess or abdominal abscess, or whether colonoscopy with surveillance biopsies was required to exclude mucosal dysplasia of the colon, before study entry was left to the clinical discretion of individual investigators. The institutional review board at each center approved the study, and all participants gave written informed consent.

\section{Concomitant Therapy}

Patients receiving corticosteroids for at least 4 weeks with a stable dose for at least 2 weeks were eligible, whereas patients in whom corticosteroids were discontinued within 2 weeks were not eligible. Patients receiving stable doses of azathioprine or 6-mercaptopurine for at least 12 weeks, or methotrexate or mycophenolate mofetil for at least 8 weeks, were eligible. Patients who had received infliximab within 4 weeks or cyclosporine within 8 weeks were not eligible. $\mathrm{Pa}$ tients who had ever received tacrolimus were not eligible (to avoid enrolling patients who had previously not responded to treatment with tacrolimus). Continued treatment with oral or rectal 5-aminosalicylates, rectal corticosteroids, and oral antibiotics was allowed, provided the dose had been stable for at least 2 weeks. Treatment with antidiarrheals (loperamide, diphenoxylate, opiates) was also allowed. No other medications for Crohn's disease were permitted. No patient had received investigational therapies within 28 days preceding randomization. Nutritional therapy (parenteral nutrition or enteral nutrition with elemental or semielemental diets) was discontinued at the screening visit and was not permitted during the trial. Any setons were removed at the screening visit.

\section{Study Medication}

Tacrolimus was initiated at a dose of $0.1 \mathrm{mg} / \mathrm{kg}$ twice daily and adjusted to a whole blood concentration of 10-20 $\mathrm{ng} / \mathrm{mL}$. The dose was selected based on studies in patients undergoing organ transplantation and 1 uncontrolled pilot study in patients with perianal Crohn's disease. ${ }^{19,21}$ Commercial tacrolimus in the form of 1-mg or 5-mg capsules (Fujisawa Ireland, Killorglin, County Kerry, Ireland; distributed by $\mathrm{Fu}-$ jisawa USA, Deerfield, IL) was overencapsulated with unlabeled red capsules by the Investigational Pharmacy Service at the Mayo Clinic Pharmacy (Rochester, MN). The Investigational Pharmacy Service prepared placebo capsules by filling the same capsules with talc (Amend Drug and Chemical
Company, Inc., Irvington, NJ, and Spectrum Chemical Manufacturing Corporation, Gardena, CA). The resulting tacrolimus and placebo $1-\mathrm{mg}$ and 5-mg capsules were identical in size and color.

\section{Study Design}

The 10-week study was a randomized, double-blind, placebo-controlled trial performed by the Crohn's and Colitis Foundation of America (CCFA) Clinical Alliance. The CCFA is a nonprofit foundation (New York, NY), and the CCFA Clinical Alliance is a group of academic medical centers, multispecialty group practices, and private practices that conduct clinical trials in inflammatory bowel disease. Eighteen centers in the United States and Canada participated in this study (Appendix 1). After a 1-week screening period, eligible patients were randomized centrally to tacrolimus or placebo in a permuted block design of 4 to ensure balanced treatment groups. The randomization schedule was generated by a statistician at the data management center for the CCFA Clinical Alliance located at the University of North Carolina (Chapel Hill, NC), and patients were assigned to a treatment group according to the schedule maintained by the Investigational Pharmacy Service of the Mayo Clinic Pharmacy.

At entry (screening visit), each patient's demographic characteristics, medical history, and current medications were recorded. Disease activity was assessed at the baseline (randomization) visit and after 2, 6, and 10 weeks. Patients recorded on diary cards the frequency of loose stools, the extent of their abdominal pain, and their general well-being during the 7 days before each visit. At each visit, a physical examination, fistula evaluation, quality-of-life assessment, and laboratory tests were conducted and patients were asked whether any adverse events had occurred.

Patients were assessed at each visit for the presence of open and actively draining perianal or enterocutaneous fistulas (defined as open fistulas with either spontaneous drainage or the ability to express drainage with gentle compression). ${ }^{14} \mathrm{~A}$ fistula diagram was completed for each patient at baseline, and every particular fistula that was open and draining was numbered. At subsequent visits, each of these numbered fistulas was evaluated and classified as being open or closed. Fistula closure was defined as the absence of drainage (spontaneous or with gentle compression) maintained for at least 4 weeks. The disease-specific instrument for measuring the activity of perianal fistulas, the perianal disease activity index, ${ }^{22}$ was not used to assess efficacy because there was no information available indicating what perianal disease activity index score constitutes fistula remission and what change in the perianal disease activity index score constitutes fistula improvement. ${ }^{23}$ Clinical disease activity was assessed with the Crohn's Disease Activity Index (CDAI); scores $<150$ points indicate clinical remission, scores of 150-219 indicate mildly active disease, scores of $220-450$ indicate moderately active disease, and scores $>450$ indicate severely active disease. ${ }^{23,24}$ The CDAI was primarily designed to measure the activity of luminal disease, and the presence of actively draining fistulas contributes only 20 points 
to the total score. ${ }^{23}$ Disease-specific health-related quality of life was assessed with the Inflammatory Bowel Disease Questionnaire (IBDQ); scores $<170$ points indicate clinically active disease, and scores $\geq 170$ points indicate clinically inactive disease. $^{25}$ All laboratory tests were performed at a central laboratory (Mayo Medical Laboratories, Rochester, MN). Blood and urine samples were taken for hematologic and biochemical assessments (including serum creatinine and glucose) and urinalysis. Measurement of whole blood tacrolimus was performed using liquid chromatography with detection by mass spectrometry. ${ }^{26,27}$

A study coordinator at the data management center reviewed the results of the whole blood tacrolimus and serum creatinine assays daily and made dose adjustments for tacrolimus using the following guidelines: (1) tacrolimus whole blood concentration $<5 \mathrm{ng} / \mathrm{mL}, 35 \%$ dose increase; (2) tacrolimus whole blood concentration 5-9 $\mathrm{ng} / \mathrm{mL}, 20 \%$ dose increase; (3) tacrolimus whole blood concentration 21-30 ng/ $\mathrm{mL}, 20 \%$ dose decrease; (4) tacrolimus whole blood concentration $>30 \mathrm{ng} / \mathrm{mL}, 35 \%$ dose decrease; (5) serum creatinine values $>1.5 \mathrm{mg} / \mathrm{dL}(>132 \mu \mathrm{mol} / \mathrm{L}), 35 \%$ dose decrease. Every time a change in drug dose was made in a patient receiving tacrolimus, a patient receiving placebo was randomly selected and the same dose change was made. The study coordinator had no contact with study investigators and did not evaluate outcomes. Dose adjustments for other adverse events including infection, hypertension, headaches, tremor, paresthesias, insomnia, diarrhea, nausea, vomiting, hyperkalemia, and hyperglycemia were handled by investigators on a case-by-case basis, taking into account the severity of the adverse event. All adverse events were classified and graded according to Costart dictionary criteria. ${ }^{28}$

\section{Outcomes and Statistical Analysis}

The intention-to-treat population included all patients who were evaluated at the screening and baseline visits and who received at least one dose of study medication. The sole primary outcome measure, as specified in the study protocol before initiating the study, was fistula improvement defined as closure of $\geq 50 \%$ of particular fistulas that were open and actively draining at baseline and maintenance of that closure for at least 4 weeks. ${ }^{14}$ The secondary outcome measures that were specified in the study protocol before initiating the study were as follows: (1) fistula remission defined as closure of all particular fistulas that were open and actively draining at baseline and maintenance of that closure for at least 4 weeks, ${ }^{14}$ (2) the mean CDAI scores at each visit, ${ }^{24}$ (3) the mean IBDQ scores at each visit, ${ }^{25}$ (4) adverse events at each visit, and (5) whole blood tacrolimus concentrations at each visit.

The rates of fistula improvement, fistula remission, and adverse events were compared using Fisher exact test. Patients who withdrew from the study prematurely because of deterioration in Crohn's disease or failure to improve were not assessed for fistula closure after withdrawal and were considered to have failed to show fistula improvement or fistula remission. All patients in the intention-to-treat population were included in these calculations. Nonparametric analysis (Wilcoxon rank sum test) was used to compare the CDAI scores, IBDQ scores, and whole blood tacrolimus concentrations. We performed exploratory comparisons to determine the impact of fistula location, number of baseline fistulas, concomitant therapy with azathioprine or 6-mercaptopurine, and antecedent therapy with infliximab on fistula improvement (Fisher exact test). We also formally performed tests for confounding by creating logistic regression models in which we added terms for a limited number of clinically sensible potential confounders: concomitant therapy with azathioprine or 6-mercaptopurine, concomitant therapy with antibiotics, antecedent therapy with infliximab, and treatment center (1-2 patients randomized at a center vs. 3-10 patients randomized at a center). We specifically sought to determine whether the addition of these terms changed the parameter estimate for the odds ratio of tacrolimus-treated patients experiencing fistula improvement compared with placebo-treated patients experiencing fistula improvement by more than $10 \%$. Items were added individually and then jointly. If there was less than a $10 \%$ change, we rejected the item as a potential confounder and did not include the item in the final model because including unnecessary items in the model would decrease the precision of the estimate. All tests were 2 sided. $P$ values $<$ 0.05 were considered statistically significant.

\section{Sample Size}

We estimated that 24 patients were needed in each of the 2 study groups to have $80 \%$ power to detect a true difference in the proportion of patients experiencing fistula improvement (the primary outcome measure) between the 2 treatment groups, assuming fistula improvement rates of $65 \%$ with tacrolimus compared with $25 \%$ with placebo.

\section{Role of the Funding Source}

The principal investigator (W.J.S.) and the other coinvestigators designed the study, recruited the patients, managed the data, performed the statistical analysis, and wrote the manuscript detailing the results of the study. Specifically, the completed case report forms for each patient were sent by investigators to the principal investigator (W.J.S., T.J.) and then to the data management center of the CCFA Clinical Alliance, where the statistical analysis was performed (J.G., C.M., R.S.S.). The study was funded by research grants from the CCFA and Fujisawa USA. Representatives from Fujisawa USA had the opportunity to review and comment on the study design and on the manuscript, but the principal investigator made the final decisions regarding the design of the study and the content of the manuscript.

\section{Results}

Fifty-four patients were screened, of whom 48 were randomized. Of the 48 randomized patients, 26 received placebo and 22 received tacrolimus. Two patients (one from each treatment group) did not have a 
Table 1. Baseline Characteristics of the Patients

\begin{tabular}{|c|c|c|c|}
\hline Variable & Placebo $(n=25)$ & Tacrolimus $(n=21)$ & $P$ \\
\hline Male sex & $11(44)$ & $10(48)$ & 1 \\
\hline White race & $21(84)$ & $19(90)$ & 0.67 \\
\hline Mean (SD, range) age at entry $(y r)^{a}$ & $38.1(13.1,12-69)$ & $40.8(11.4,21-65)$ & 0.51 \\
\hline Mean (SD) body mass index $\left(\mathrm{kg} / \mathrm{m}^{2}\right)$ & $25.3(6.8)$ & $27.1(6.1)$ & 0.58 \\
\hline \multicolumn{4}{|l|}{ Disease site (no. of patients) ${ }^{b}$} \\
\hline Ileum & $6(26)$ & $2(11)$ & 0.36 \\
\hline Ileum and colon & $6(26)$ & $8(44)$ & \\
\hline Colon & $11(48)$ & $8(44)$ & \\
\hline Previous intestinal resection (no. of patients) & $11(44)$ & $13(62)$ & 0.25 \\
\hline Current smoker & $4(16)$ & 7 (33) & 0.30 \\
\hline \multicolumn{4}{|l|}{ Location of fistulas } \\
\hline Abdominal & $3(12)$ & $0(0)$ & 0.16 \\
\hline Perianal & $22(88)$ & $20(95)$ & \\
\hline Both & $0(0)$ & $1(5)$ & \\
\hline \multicolumn{4}{|l|}{ Number of open draining fistulas at baseline } \\
\hline 1 & $13(52)$ & $6(29)$ & 0.14 \\
\hline 2 & $5(20)$ & $7(33)$ & \\
\hline 3 & $7(28)$ & $5(24)$ & \\
\hline$>3$ & $0(0)$ & $3(14)$ & \\
\hline Mean (SD) CDAl score ${ }^{c}$ & $196(108)$ & $195(93)$ & 0.96 \\
\hline Mean (SD) IBDQ score ${ }^{d}$ & $149(36)$ & $155(35)$ & 0.57 \\
\hline \multicolumn{4}{|l|}{ Concomitant medications } \\
\hline Corticosteroids & $4(16)$ & $5(24)$ & 0.71 \\
\hline Azathioprine or 6-mercaptopurine & $14(56)$ & $13(62)$ & 0.77 \\
\hline Methotrexate & $0(0)$ & $0(0)$ & 1 \\
\hline Antibiotics $^{e}$ & $19(76)$ & $13(62)$ & 0.35 \\
\hline 5-Aminosalicylates ${ }^{f}$ & $10(40)$ & $9(43)$ & 1 \\
\hline Prior treatment with infliximab at any time & $14(56)$ & $15(71)$ & 0.36 \\
\hline
\end{tabular}

NOTE. Data are expressed as number (percent) unless otherwise indicated.

apatients aged younger than 18 years is 2 for placebo and 0 for tacrolimus.

${ }^{b} \mathrm{n}=23$ for placebo and $\mathrm{n}=18$ for tacrolimus due to missing data.

'Scores $<150$ points indicate clinical remission, scores of 150-219 indicate mildly active disease, scores of 220-450 indicate moderately active disease, and scores $>450$ indicate severely active disease.

${ }^{d}$ Scores $<170$ points indicate clinically active disease, and scores $\geq 170$ points indicate clinically inactive disease.

eCiprofloxacin, metronidazole, trimethoprim/sulfamethoxazole, penicillin, ampicillin/clavulanic acid, azithromycin, clarithromycin, or doxycycline.

${ }^{\prime}$ Mesalamine or sulfasalazine.

baseline fistula assessment at week 0 and were excluded from the analysis. The remaining 46 patients comprised the intention-to-treat population. The baseline characteristics of the 2 groups of patients were similar (Table 1). The trial profile is shown in Figure 1.

\section{Clinical Effectiveness}

The rate of fistula improvement was significantly greater in patients treated with tacrolimus ( 9 of 21 [43\%]) compared with placebo (2 of 25 [8\%]) $(P=$ $0.01)$. The rates of fistula remission were similar in patients treated with tacrolimus (2 of 21 [10\%]) compared with placebo ( 2 of $25[8 \%])(P=1.0)$. The mean CDAI and IBDQ scores were similar throughout the study (data not shown). Some exploratory comparisons were made to determine the impact of disease location, number of baseline fistulas, and concomitant therapy on fistula improvement. Forty-two patients had only perianal fistulas; 9 of 20 tacrolimus-treated patients (45\%) had fistula improvement compared with 2 of 22 placebotreated patients $(9 \%)(P=0.01)$. None of 3 patients $(0 \%)$ with abdominal fistulas treated with placebo had fistula improvement, and 0 of 1 patient $(0 \%)$ with both perianal and abdominal fistulas treated with tacrolimus improved. Of the patients with 1 fistula at baseline, 1 of 13 patients $(8 \%)$ treated with placebo improved compared with 1 of $6(17 \%)$ treated with tacrolimus $(P=$ 1.0). Of the patients with 2 fistulas at baseline, 0 of 5 $(0 \%)$ treated with placebo improved compared with 4 of $7(57 \%)$ treated with tacrolimus $(P=0.08)$. Of the patients with 3 fistulas at baseline, 1 of $7(14 \%)$ treated with placebo improved compared with 2 of $5(40 \%)$ treated with tacrolimus $(P=0.52)$. Of the patients with $>3$ fistulas at baseline, none were treated with placebo and 2 of $3(67 \%)$ treated with tacrolimus improved. Twenty-seven patients were receiving concomitant therapy with azathioprine or 6-mercaptopurine. Among tacrolimus-treated patients, the rates of fistula improve- 


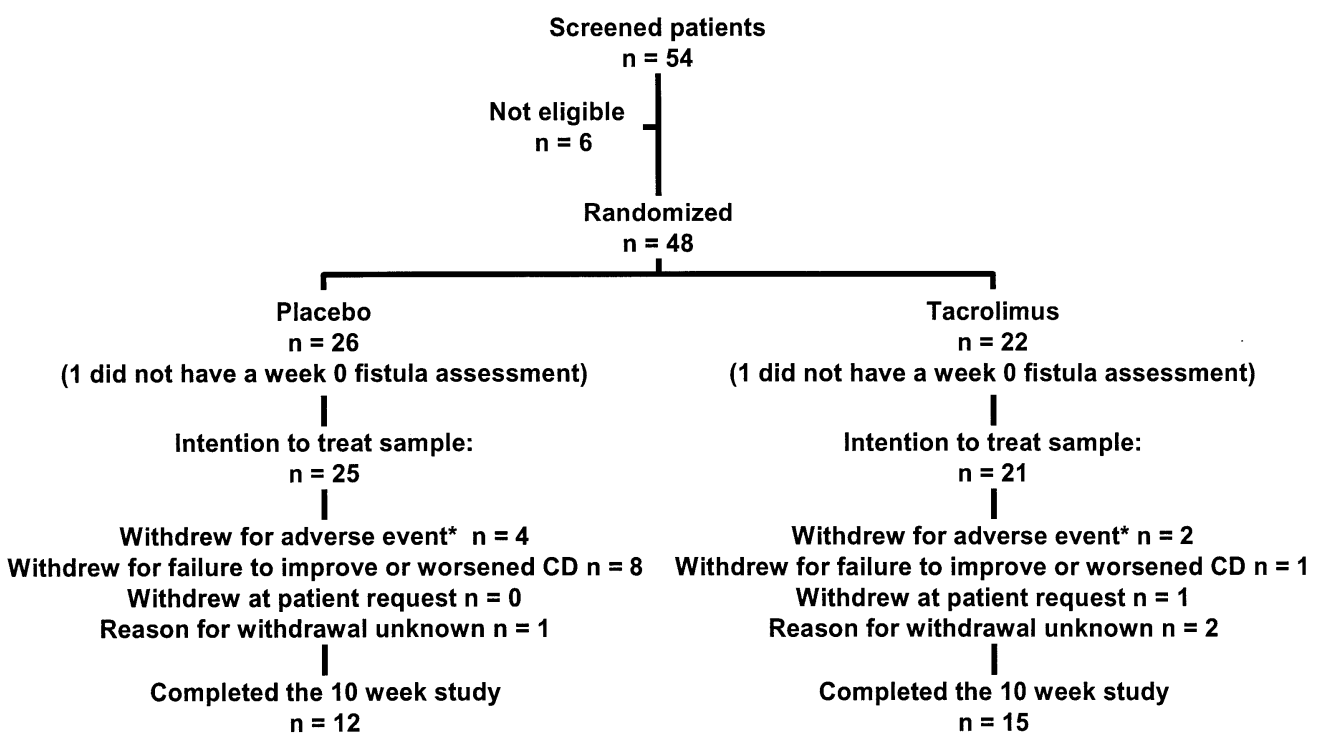

Figure 1. Trial profile. For patients who did not complete the study for more than one reason, only one reason is included in the table, according to the following hierarchy: lost to follow-up > non-Crohn's disease-associated adverse event $>$ deterioration of Crohn's disease or failure to improve $>$ patient request $>$ reason for withdrawal not available. *For placebo-treated patients, the adverse events that led to withdrawal from the study were new perianal abscess (1 patient), nephrotoxicity and hepatotoxicity (1 patient), upper respiratory infection (1 patient), and no reason specified (1 patient). For tacrolimus-treated patients, the adverse events that led to withdrawal from the study were a combination of headaches, dizziness, blurred vision, paresthesias, purpura, pruritus, and insomnia (1 patient), and no reason specified (1 patient).

ment were similar in patients receiving concomitant therapy with azathioprine or 6-mercaptopurine ( 5 of 13 [38\%]) and patients not receiving these agents ( 4 of 8 [50\%]) $(P=0.31$; Fisher exact test). Among placebotreated patients, the rates of fistula improvement were similar in patients receiving concomitant therapy with azathioprine or 6-mercaptopurine (2 of 14 [14\%]) and patients not receiving these agents ( 0 of $11[0 \%])(P=$ $0.30)$. Twenty-nine patients had previously been treated with infliximab. Among tacrolimus-treated patients, the rates of fistula improvement were similar in patients previously treated with infliximab (7 of 15 [47\%]) and infliximab-naive patients ( 2 of $6[33 \%])(P=0.66$; Fisher exact test). Likewise, among placebo-treated patients, the rates of fistula improvement were similar in patients previously treated with infliximab ( 0 of 14 [0\%]) and infliximab-naive patients (2 of 11 [18\%]) $(P=0.18)$. The odds ratio (95\% confidence interval) of tacrolimus-treated patients experiencing fistula improvement compared with placebo-treated patients experiencing fistula improvement was $8.62(1.60,46.45)(P=$ $0.01)$. The odds ratios after the addition of the following potential confounders were as follows: concomitant azathioprine or 6-mercaptopurine, $8.56(1.59,46.14)(P=$ $0.01)$; concomitant antibiotics, $8.15(1.44,46.04)(P=$ $0.02)$; antecedent infliximab, $9.23(1.65,51.72)(P=$ $0.01)$; treatment center, $8.92(1.63,48.64)(P=0.01)$; all 4 potential confounders combined, $7.74(1.28,46.80)$ $(P=0.03)$. None of the parameter estimates changed by more than $10 \%$, suggesting that none of the candidate items individually or in combination were confounders.

\section{Tacrolimus Dosing and Whole Blood Concentrations}

The mean tacrolimus doses and whole blood tacrolimus concentrations at each visit for patients in the tacrolimus group are shown in Table 2. Nineteen of 21 patients $(90 \%)$ treated with tacrolimus required at least 1 dose change due to whole blood concentrations above or below the target range and/or an increased serum creatinine concentration to a value $\geq 1.5 \mathrm{mg} / \mathrm{dL}(>132$ $\mu \mathrm{mol} / \mathrm{L})$ and/or other dose-limiting adverse events. The mean whole blood tacrolimus concentrations in patients treated with tacrolimus who had fistula improvement

Table 2. Tacrolimus Dosing and Whole Blood Concentrations

\begin{tabular}{lcc}
\hline Week of visit & $\begin{array}{c}\text { Tacrolimus dose } \\
\left(\mathrm{mg} \cdot \mathrm{kg}^{-1} \cdot \mathrm{day}^{-1}\right)\end{array}$ & $\begin{array}{c}\text { Whole blood tacrolimus } \\
\text { concentrations }(\mathrm{ng} / \mathrm{mL})\end{array}$ \\
\hline 0 (baseline) & $0.202 \pm 0.010$ & $0.0 \pm 0.0$ \\
1 & $0.186 \pm 0.027$ & $22.5 \pm 13.4$ \\
2 & $0.169 \pm 0.045$ & $15.3 \pm 8.3$ \\
3 & $0.166 \pm 0.055$ & $15.3 \pm 8.5$ \\
4 & $0.168 \pm 0.068$ & $14.3 \pm 8.6$ \\
6 & $0.164 \pm 0.091$ & $13.0 \pm 9.7$ \\
8 & $0.155 \pm 0.097$ & $10.5 \pm 6.3$ \\
10 & $0.155 \pm 0.110$ & $8.3 \pm 6.9$ \\
\hline
\end{tabular}

NOTE. Data are expressed as mean \pm SD . 
Table 3. Adverse Events in the 2 Treatment Groups

\begin{tabular}{|c|c|c|c|}
\hline Variable & $\begin{array}{l}\text { Placebo group } \\
\quad(\mathrm{n}=25)\end{array}$ & $\begin{array}{l}\text { Tacrolimus group } \\
\qquad(\mathrm{n}=21)\end{array}$ & $P$ \\
\hline Mean (SD) no. of adverse events per patient & $2.3(2.3)$ & $5.2(4.2)$ & 0.009 \\
\hline No. $(\%, 95 \% \mathrm{Cl})$ of patients with adverse events & $19(76,57-89)$ & $20(95,79-100)$ & 0.11 \\
\hline No. $(\%, 95 \% \mathrm{Cl})$ of patients with serious adverse events & $0(0,0-12)$ & $1(5,0-21)$ & 0.46 \\
\hline No. $(\%, 95 \% \mathrm{Cl})$ of patients with severe adverse events & $2(8,1-24)$ & $6(29,13-51)$ & 0.12 \\
\hline \multicolumn{4}{|c|}{$\begin{array}{l}\text { No. (\%, } 95 \% \mathrm{Cl} \text { ) of adverse events occurring in } \geq 10 \% \text { of patients in } \\
\text { at least one of the treatment groups }\end{array}$} \\
\hline Abdominal pain & $2(8,1-24)$ & $3(14,4-34)$ & 0.65 \\
\hline Asthenia & $1(4,0-19)$ & $2(10,2-28)$ & 0.58 \\
\hline Creatinine level increased & $0(0,0-12)$ & $8(38,20-60)$ & 0.008 \\
\hline Diarrhea & $3(12,3-30)$ & $4(19,7-40)$ & 0.69 \\
\hline Dizziness & $0(0,0-12)$ & $2(10,2-28)$ & 0.20 \\
\hline Esophagitis & $1(4,0-19)$ & $2(10,2-28)$ & 0.58 \\
\hline Headache & $3(12,3-30)$ & $10(48,28-72)$ & 0.01 \\
\hline Hyperkalemia & $0(0,0-12)$ & $2(10,2-28)$ & 0.20 \\
\hline Insomnia & $0(0,0-12)$ & $6(29,13-51)$ & 0.006 \\
\hline Leg cramps & $0(0,0-12)$ & $5(24,10-46)$ & 0.01 \\
\hline Leukocytosis & $0(0,0-12)$ & $2(10,2-28)$ & 0.20 \\
\hline Nausea and/or vomiting & $4(16,6-34)$ & $6(29,13-51)$ & 0.47 \\
\hline Paresthesias & $1(4,0-19)$ & $12(57,34-79)$ & $<0.001$ \\
\hline Peripheral edema & $0(0,0-12)$ & $2(10,2-28)$ & 0.20 \\
\hline Pruritus & $1(4,0-19)$ & $4(19,7-40)$ & 0.16 \\
\hline Rectal disorder & $5(20,8-38)$ & $3(14,4-34)$ & 0.71 \\
\hline Rhinitis & $3(12,3-30)$ & $2(10,2-28)$ & 1.00 \\
\hline Thinking abnormal & $0(0,0-12)$ & $2(10,2-28)$ & 0.20 \\
\hline Tremor & $0(0,0-12)$ & $6(29,13-51)$ & 0.006 \\
\hline Other & $11(44,24-66)$ & $11(52,28-72)$ & 0.77 \\
\hline
\end{tabular}

and those who did not were the same $(13.7 \mathrm{ng} / \mathrm{mL})(P=$ 0.92; Mann-Whitney test).

\section{Adverse Events}

The mean number of adverse events per patient was significantly greater in the tacrolimus group (Table 3). The proportions of patients experiencing any adverse events, severe adverse events, and serious adverse events were similar in the 2 treatment groups (Table 3). The adverse events that occurred at a frequency $\geq 10 \%$ are also shown in Table 3. There were significant differences between the 2 treatment groups in the proportions of patients with adverse events known to be associated with tacrolimus (increased serum creatinine level, headache, insomnia, paresthesias, and tremor) (Table 3). Eight of 21 patients (38\%) treated with tacrolimus had an increase in serum creatinine level from baseline to a value $\geq 1.5 \mathrm{mg} / \mathrm{dL}(>132 \mu \mathrm{mol} / \mathrm{L})$ (designated before the study as nephrotoxicity requiring tacrolimus dose reduction) compared with 0 of 25 placebo-treated patients $(0 \%)(P=0.008)$. Two patients withdrew from the study prematurely because of tacrolimus-associated adverse events (Figure 1). All other adverse events that occurred in tacrolimus-treated patients were successfully managed with dose reduction. No other differences between the 2 treatment groups in the proportions of patients with individual types of adverse events were significant, and there were no other clinically important differences between the treatment groups with respect to changes in laboratory assessments.

\section{Discussion}

We found that oral tacrolimus $0.2 \mathrm{mg} \cdot \mathrm{kg}^{-1}$. day $^{-1}$ is effective for inducing improvement of fistula drainage, but not fistula remission, in patients with Crohn's disease. The end point of improvement in fistula drainage, which has been accepted as clinically meaningful by regulatory authorities in North America and Europe, may be more sensitive to smaller degrees of efficacy than complete fistula remission. Nevertheless, some experts believe that complete fistula remission may be a more clinically relevant end point. ${ }^{23}$ In our study, the rate of complete fistula remission was low in both treatment groups and the difference was not significant. Whether the failure to achieve a significantly higher rate of complete fistula remission was due to a relatively weak treatment effect or due to inadequate statistical power to detect meaningful differences between treatment groups for this secondary end point is uncertain. A substantial proportion of the patients had previously been treated with infliximab therapy or were receiving concomitant treatment with azathioprine or 6-mercaptopurine. We assessed the possible influence of these interventions on 
the primary outcome by means of a logistic regression model and found no evidence of a significant confounding effect. It should be noted that we found no evidence of efficacy in patients with abdominal fistulas, and thus the results of our study apply primarily to patients with perianal fistulas. The patients in the placebo and tacrolimus treatment groups in our study had relatively low mean baseline CDAI scores (196 and 195) and relatively high mean baseline IBDQ scores (149 and 155), indicating relatively low overall Crohn's disease activity; these parameters did not change significantly during treatment with tacrolimus. Our results showing relatively low CDAI scores in patients with actively draining fistulas, which are similar to those reported in a controlled trial of infliximab for the treatment of fistulas, ${ }^{14}$ are not surprising given that the CDAI and IBDQ instruments were not designed to assess the type of morbidity that patients with actively draining fistulas experience. ,, $23-25^{-}$

The limitations of this study include inadequate statistical power to assess the effectiveness of tacrolimus for the secondary end point of fistula remission, lack of a range of dose groups to determine the lowest effective dose of tacrolimus, lack of information regarding the durability of fistula improvement after tacrolimus is discontinued, and lack of information regarding the long-term effects of tacrolimus therapy on renal function. Now that we have shown that tacrolimus is effective for treating Crohn's disease fistulas, additional studies can be designed to address these unanswered questions. In addition, we did not perform pretreatment evaluation with examination under anesthesia, anorectal ultrasonography, or pelvic magnetic resonance imaging in patients entering this study. ${ }^{29}$ Other pilot studies in patients treated with infliximab have suggested that such imaging procedures may improve treatment outcomes. ${ }^{30,31}$

The initial tacrolimus dose of $0.2 \mathrm{mg} \cdot \mathrm{kg}^{-1} \cdot \mathrm{day}^{-1}$ resulted in a mean whole blood tacrolimus concentration at week 1 of $22.5 \mathrm{ng} / \mathrm{mL}$ (target range for this study, $10-20 \mathrm{ng} / \mathrm{mL}$; published therapeutic range for transplantation, 3-20 $\mathrm{ng} / \mathrm{mL}$ ). Nineteen of 21 tacrolimustreated patients $(90 \%)$ required dose reduction because of either high tacrolimus blood concentrations or adverse events (see following text). The mean final dose of tacrolimus at week 10 was $0.16 \mathrm{mg} \cdot \mathrm{kg}^{-1} \cdot \mathrm{day}^{-1}$, and the mean final tacrolimus blood concentration was $8.3 \mathrm{ng} /$ $\mathrm{mL}$. These results suggest that an initial tacrolimus dose of $0.2 \mathrm{mg} \cdot \mathrm{kg}^{-1} \cdot$ day $^{-1}$, adjusted to a target blood concentration range and for toxicity, is the maximal tolerated dose for the treatment of Crohn's disease. The lack of a significant correlation between fistula improvement and tacrolimus blood concentrations is likely a result of our administering a single dose of tacrolimus adjusted to a relatively narrow range of high tacrolimus blood concentrations. An uncontrolled pilot study of low-dose tacrolimus (2-4 mg/day) reported a beneficial effect despite low tacrolimus blood concentrations $(<10$ $\mathrm{ng} / \mathrm{mL}$ ) in patients with rheumatoid arthritis. ${ }^{32}$ Because most adverse events from tacrolimus in our study were dose related, the efficacy of low-dose tacrolimus (0.05$0.15 \mathrm{mg} \cdot \mathrm{kg}^{-1} \cdot \mathrm{day}^{-1}$ ) adjusted to lower target blood levels $(3-10 \mathrm{ng} / \mathrm{mL})$ for the treatment of fistulas in patients with Crohn's disease should be determined.

The mean number of adverse events per patient was significantly increased in patients treated with tacrolimus. This difference between treatment groups was almost entirely due to an increased frequency of adverse events known to be associated with tacrolimus, including increased serum creatinine level, headache, insomnia, paresthesias, and tremor. ${ }^{21}$ These adverse events related to tacrolimus were for the most part successfully managed with dose reduction. The proportions of patients experiencing any adverse event and severe and serious adverse events were not significantly increased in the tacrolimus group, and only 2 tacrolimus-treated patients $(10 \%)$ withdrew from the trial prematurely due to adverse events (compared to 4 placebo-treated patients [16\%]). No patients developed serious infections, sepsis, or opportunistic infections. The most significant adverse event that occurred in patients treated with tacrolimus was nephrotoxicity. Eight of 21 patients $(38 \%)$ treated with tacrolimus had an increase in serum creatinine level from baseline to a value $\geq 1.5 \mathrm{mg} / \mathrm{dL}(>132 \mu \mathrm{mol} / \mathrm{L})$, indicating an increase from baseline serum creatinine level of $>30 \%$. It is possible that more sensitive measures of renal function such as creatinine clearance, inulin clearance, or iothalamate clearance might have shown an even greater rate of nephrotoxicity. In patients treated with another calcineurin inhibitor, cyclosporine, an increased frequency of striped interstitial nephritis and arteriolar alterations on renal biopsy has been associated with high-dose cyclosporine and an increase in serum creatinine level $>30 \%$ above the baseline value. ${ }^{33}$ Similar histologic lesions have been observed in patients with tacrolimus-associated nephrotoxicity, ${ }^{34}$ and it is reasonable to speculate that high-dose tacrolimus therapy or a significant increase in serum creatinine level from baseline in tacrolimus-treated patients may lead to important renal pathology. These histologic changes are believed to be irreversible. Concern about potential nephrotoxicity will likely limit the use of high-dose tacrolimus to patients with Crohn's disease fistulas refractory to other therapies, including azathioprine, 6-mercaptopurine, and 
infliximab, and emphasizes the need for a study to explore the safety and effectiveness of lower doses of tacrolimus.

Finally, the issue of which patients are appropriate candidates for tacrolimus therapy of Crohn's disease fistulas must be addressed. In our study, $63 \%$ of patients had previously been treated with infliximab, and 56\%$62 \%$ were receiving concomitant therapy with azathioprine or 6-mercaptopurine. A natural history study of patients with perianal Crohn's disease suggested that most patients are adequately treated with minor perianal surgical procedures such as incision and drainage of abscesses or fistulotomy or else medical therapy with antibiotics such as metronidazole or ciprofloxacin. ${ }^{4} \mathrm{~A}$ recently published treatment algorithm suggesting that therapy with tacrolimus be considered in patients failing therapy with azathioprine or 6-mercaptopurine and infliximab as an alternative to proctectomy appears to be justified given the results of our study. ${ }^{5}$ However, because of the potential for nephrotoxicity from high-dose tacrolimus and because complete fistula remission was not achieved, this agent should probably be reserved for patients who fail or are intolerant to all other therapies, including antibiotics, azathioprine and 6-mecaptopurine, and infliximab. The observed rates of complete fistula closure with tacrolimus are numerically lower in our study than those observed with infliximab. ${ }^{14}$ Although such comparisons across clinical trials should be undertaken with great caution due to lack of randomization, it appears that tacrolimus may be less effective than infliximab for the treatment of Crohn's disease fistulas. The toxicity profile of cyclosporine at doses that are potentially effective for the treatment of Crohn's disease fistulas is similar to that observed with tacrolimus, ${ }^{10-12,35}$ and there are no controlled data regarding the effectiveness of cyclosporine for this indication. Because there are now controlled trial data showing that tacrolimus is effective for Crohn's disease fistulas, tacrolimus, in preference to cyclosporine, may be considered in patients who have failed antibiotics, azathioprine or 6-mercaptopurine, and infliximab. Given the lack of data in patients with abdominal fistulas, the use of tacrolimus should probably be confined to patients with perianal fistulas.

In conclusion, oral tacrolimus at an initial dose of 0.2 $\mathrm{mg} \cdot \mathrm{kg}^{-1} \cdot \mathrm{day}^{-1}$ is effective for fistula improvement, but not fistula remission, in patients with perianal Crohn's disease. Adverse events, which occurred frequently in tacrolimus-treated patients, could be managed in most instances with dose reduction. The effectiveness of lower doses of tacrolimus should be determined.

\section{Appendix 1. CCFA Clinical Alliance Investigators}

Stephen Hanauer, M.D., University of Chicago, Chicago, Illinois; Daniel Present, M.D., and Lloyd Mayer, M.D., Mount Sinai School of Medicine, New York, New York; William J. Sandborn, M.D., Edward V. Loftus, M.D., William J. Tremaine, M.D., Therese Johnson, R.N., and Resa Jeche, Mayo Clinic, Rochester, Minnesota; Lori Kam, M.D., Stephan Targan, M.D., and Eric Vasiliauskas, M.D., Cedars Sinai Medical Center, Los Angeles, California; Douglas C. Wolf, M.D., and Bruce Salzberg, M.D., Atlanta Gastroenterology Associates, Atlanta, Georgia; Brian Feagan, M.D., University of Western Ontario, London, Ontario, Canada; Lloyd Sutherland, M.D., and Remo Panaccione, M.D., University of Calgary, Calgary, Alberta, Canada; Jeffery Katz, M.D., Case Western Reserve University, Cleveland, Ohio; Ronald Fogel, M.D., Henry Ford Hospital, Detroit, Michigan; Robert Sandler, M.D., Joseph Galanko, Ph.D., Kim Isaacs, M.D., Ph.D., Christopher Martin, M.P.H., R. Balfour Sartor, M.D., Ella Akin, and M. Kate Sirah, University of North Carolina Chapel Hill, Chapel Hill, North Carolina; Edward Hoffenberg, M.D., The Children's Hospital, Denver, Colorado; John Valentine, M.D., University of Florida, Gainesville, Florida; Jonathan Leighton, M.D., Mayo Clinic Scottsdale, Scottsdale, Arizona; John R. Cangemi, M.D., Mayo Clinic Jacksonville, Jacksonville, Florida; Ben Dolin, Gastroenterology Ltd., Peoria, Illinois; Eugene Greenberg, M.D., Carle Clinic Association, Urbana, Illinois; Mark Reichelderfer, M.D., University of Wisconsin, Madison, Wisconsin; Bruce E. Sands, Harvard Medical School, Massachusetts General Hospital, Boston, Massachusetts; and James Markowitz, M.D., North Shore University Hospital, Manhasset, New York

\section{References}

1. Farmer RG, Hawk WA, Turnbull RB Jr. Clinical patterns in Crohn's disease: a statistical study of 615 cases. Gastroenterology 1975;68:627-635.

2. Rankin GB, Watts HD, Melnyk CS, Kelley ML Jr. National Cooperative Crohn's Disease Study: extraintestinal manifestations and perianal complications. Gastroenterology 1979;77:914-920.

3. Hellers G, Bergstrand O, Ewerth S, Holmstrom B. Occurrence and outcome after primary treatment of anal fistulae in Crohn's disease. Gut 1980;21:525-527.

4. Schwartz DA, Loftus EV Jr, Tremaine WJ, Panaccione R, Harmsen WS, Zinsmeister AR, Sandborn WJ. The natural history of fistulizing Crohn's disease in Olmsted County, Minnesota. Gastroenterology 2002;122:875-880.

5. Schwartz DA, Pemberton JH, Sandborn WJ. Diagnosis and treatment of perianal fistulas in Crohn disease. Ann Intern Med 2001; 135:906-918.

6. Lichtenstein GR. Treatment of fistulizing Crohn's disease. Gastroenterology 2000;119:1132-1147.

7. Bernstein LH, Frank MS, Brandt LJ, Boley SJ. Healing of perineal Crohn's disease with metronidazole. Gastroenterology 1980;79: 357-365.

8. Brandt LJ, Bernstein LH, Boley SJ, Frank MS. Metronidazole therapy for perineal Crohn's disease: a follow-up study. Gastroenterology 1982;83:383-387. 
9. Korelitz BI, Present DH. Favorable effect of 6-mercaptopurine on fistulae of Crohn's disease. Dig Dis Sci 1985;30:58-64.

10. Hanauer SB, Smith MB. Rapid closure of Crohn's disease fistulas with continuous intravenous cyclosporin A. Am J Gastroenterol 1993;88:646-649.

11. Present DH, Lichtiger S. Efficacy of cyclosporine in treatment of fistula of Crohn's disease. Dig Dis Sci 1994;39:374-380.

12. Egan LJ, Sandborn WJ, Tremaine WJ. Clinical outcome following treatment of refractory inflammatory and fistulizing Crohn's disease with intravenous cyclosporine. Am J Gastroenterol 1998; 93:442-448.

13. Pearson DC, May GR, Fick GH, Sutherland LR. Azathioprine and 6-mercaptopurine in Crohn disease. A meta-analysis. Ann Intern Med 1995;123:132-142.

14. Present DH, Rutgeerts P, Targan S, Hanauer SB, Mayer L, van Hogezand RA, Podolsky DK, Sands BE, Braakman T, DeWoody KL, Schaible TF, van Deventer SJ. Infliximab for the treatment of fistulas in patients with Crohn's disease. N Engl J Med 1999; 340:1398-1405.

15. Liu J, Farmer JD Jr, Lane WS, Friedman J, Weissman I, Schreiber $\mathrm{SL}$. Calcineurin is a common target of cyclophilin-cyclosporin A and FKBP- FK506 complexes. Cell 1991;66:807-815.

16. Flanagan WM, Corthesy B, Bram RJ, Crabtree GR. Nuclear association of a T-cell transcription factor blocked by FK-506 and cyclosporin A. Nature 1991;352:803-807.

17. Thomson AW, Carroll PB, McCauley J, Woo J, Abu-Elmagd K, Starzl TE, Van Thiel DH. FK 506: a novel immunosuppressant for treatment of autoimmune disease. Rationale and preliminary clinical experience at the University of Pittsburgh. Springer Semin Immunopathol 1993;14:323-344.

18. Sandborn WJ. Preliminary report on the use of oral tacrolimus (FK506) in the treatment of complicated proximal small bowel and fistulizing Crohn's disease. Am J Gastroenterol 1997;92: 876-879.

19. Lowry PW, Weaver AL, Tremaine WJ, Sandborn WJ. Combination therapy with oral tacrolimus (FK506) and azathioprine or 6-mercaptopurine for treatment-refractory Crohn's disease perianal fistulae. Inflamm Bowel Dis 1999;5:239-245.

20. Ierardi E, Principi M, Rendina M, Francavilla R, Ingrosso M, Pisani A, Amoruso A, Panella C, Francavilla A. Oral tacrolimus (FK 506) in Crohn's disease complicated by fistulae of the perineum. J Clin Gastroenterol 2000;30:200-202.

21. Spencer CM, Goa KL, Gillis JC. Tacrolimus. An update of its pharmacology and clinical efficacy in the management of organ transplantation. Drugs 1997;54:925-975.

22. Irvine EJ. Usual therapy improves perianal Crohn's disease as measured by a new disease activity index. McMaster IBD Study Group. J Clin Gastroenterol 1995;20:27-32.

23. Sandborn WJ, Feagan BG, Hanauer SB, Lochs H, Lofberg R, Modigliani R, Present DH, Rutgeerts P, Scholmerich J, Stange EF, Sutherland LR. A review of activity indices and efficacy endpoints for clinical trials of medical therapy in adults with Crohn's disease. Gastroenterology 2002;122:512-530.

24. Best WR, Becktel JM, Singleton JW, Kern F Jr. Development of a Crohn's disease activity index. National Cooperative Crohn's Disease Study. Gastroenterology 1976;70:439-444.
25. Irvine EJ, Feagan B, Rochon J, Archambault A, Fedorak RN, Groll A, Kinnear D, Saibil F, McDonald JW. Quality of life: a valid and reliable measure of therapeutic efficacy in the treatment of inflammatory bowel disease. Canadian Crohn's Relapse Prevention Trial Study Group. Gastroenterology 1994;106:287-296.

26. Wingard JR, Nash RA, Przepiorka D, Klein JL, Weisdorf DJ, Fay JW, Zhu J, Maher RM, Fitzsimmons WE, Ratanatharathorn V. Relationship of tacrolimus (FK506) whole blood concentrations and efficacy and safety after HLA-identical sibling bone marrow transplantation. Biol Blood Marrow Transplant 1998;4:157-163.

27. Cheryk L, Hoefner D, Velosa J, Moyer T. Optimal tacrolimus steady state blood concentration for renal transplantation (abstr). Clin Chem 1999;45:A68.

28. COSTART: Coding symbols for thesaurus of adverse reaction terms. 5th ed. Department of Health and Human Services, Food and Drug Administration (FDA), 1995.

29. Schwartz DA, Wiersema MJ, Dudiak KM, Fletcher JG, Clain JE, Tremaine WJ, Zinsmeister AR, Norton ID, Boardman LA, Devine RM, Wolff BG, Young-Fadok TM, Diehl NN, Pemberton JH, Sandborn WJ. A comparison of endoscopic ultrasound, magnetic resonance imaging, and exam under anesthesia for evaluation of Crohn's perianal fistulas. Gastroenterology 2001;121:10641072.

30. Van Assche G, Vanbeckevoort D, Bielen D, Coremans G, Aerden I, Norman M, Vermeire S, Marchal G, Cornillie F, Rutgeerts P. MRI imaging of the effects of infliximab in perianal fistulizing Crohn's disease (abstr). Gastroenterology 2001;120:A68.

31. Regueiro M, Mardini H. Treatment of perianal fistulizing Crohn's disease (CD) with infliximab alone or as an adjunct to exam under anesthesia (EUA) (abstr). Gastroenterology 2002;122:A614.

32. Gremillion RB, Posever JO, Manek N, West JP, van Volen-Hoven RF. Tacrolimus (FK506) in the treatment of severe, refractory rheumatoid arthritis: initial experience in 12 patients. J Rheumatol 1999;26:2332-2336.

33. Feutren G, Mihatsch MJ. Risk factors for cyclosporine-induced nephropathy in patients with autoimmune diseases. International Kidney Biopsy Registry of Cyclosporine in Autoimmune Diseases. N Engl J Med 1992;326:1654-1660.

34. Randhawa PS, Shapiro R, Jordan ML, Starzl TE, Demetris AJ. The histopathological changes associated with allograft rejection and drug toxicity in renal transplant recipients maintained on FK506. Clinical significance and comparison with cyclosporine. Am J Surg Pathol 1993;17:60-68.

35. Sandborn WJ. A critical review of cyclosporine therapy in inflammatory bowel disease. Inflamm Bowel Dis 1995;1:48-63.

Received September 9, 2002. Accepted May 1, 2003.

Address requests for reprints to: William J. Sandborn, M.D., Mayo Clinic, 200 First Street Southwest, Rochester, Minnesota 55905. email: sandborn.william@mayo.edu; fax: (507) 266-0335.

Supported by research grants from the Crohn's and Colitis Foundation of America (New York, NY), Fujisawa USA (Deerfield, IL), and the General Clinical Research Centers program of the Division of Research Resources, National Institutes of Health (grant no. RR00046).

W.J.S. has received research support from Fujisawa USA. 